\title{
Resource Allocation for Spectrum Underlay in Cognitive Radio Networks
}

\author{
Long Bao Le, Member, IEEE, and Ekram Hossain, Senior Member, IEEE
}

\begin{abstract}
A resource allocation framework is presented for spectrum underlay in cognitive wireless networks. We consider both interference constraints for primary users and quality of service $(\mathrm{QoS})$ constraints for secondary users. Specifically, interference from secondary users to primary users is constrained to be below a tolerable limit. Also, signal to interference plus noise ratio (SINR) of each secondary user is maintained higher than a desired level for QoS insurance. We propose admission control algorithms to be used during high network load conditions which are performed jointly with power control so that QoS requirements of all admitted secondary users are satisfied while keeping the interference to primary users below the tolerable limit. If all secondary users can be supported at minimum rates, we allow them to increase their transmission rates and share the spectrum in a fair manner. We formulate the joint power/rate allocation with proportional and max-min fairness criteria as optimization problems. We show how to transform these optimization problems into a convex form so that their globally optimal solutions can be obtained. Numerical results show that the proposed admission control algorithms achieve performance very close to that of the optimal solution. Also, impacts of different system and QoS parameters on the network performance are investigated for the admission control, and rate/power allocation algorithms under different fairness criteria.
\end{abstract}

Index Terms-Cognitive radio, spectrum sharing, spectrum underlay, spectrum overlay, interference temperature limit, admission control, rate and power allocation.

\section{INTRODUCTION}

$\mathbf{T}$ HE emerging high-speed wireless access technologies and the requirements of different wireless applications are expected to create huge demand for spectral resources in the next generation wireless systems. Achieving high spectrum utilization is, therefore, one of the most critical research objectives in designing wireless communication systems today. In fact, it has been reported that current utilization of some allocated spectrum can be as low as $15 \%$ [1]. Therefore, there is an increasing interest in developing efficient methods for spectrum management and sharing which is encouraged by both industry and FCC [2]. This motivates to exploit spectrum opportunities in space, time, frequency while protecting users

Manuscript received August 10, 2007; revised October 15, 2007; accepted October 26, 2007. The associate editor coordinating the review of this paper and approving it for publication was M. Guizani. This work was supported in part by the University of Manitoba Graduate Fellowship (UMGF) and in part by a grant from the Natural Sciences and Engineering Research Council (NSERC) of Canada.

L. B. Le is with the Massachusetts Institute of Technology, USA (e-mail: longble@mit.edu).

E. Hossain is with the Department of Electrical and Computer Engineering, University of Manitoba, Winnipeg, MB, Canada R3T 5V6 (e-mail: ekram@ee.umanitoba.ca).

Digital Object Identifier 10.1109/T-WC.2008.070890 of the primary network from excessive interference due to spectrum access from secondary networks. As described in [3], users from a secondary network (who are referred to as secondary users) can access the spectrum owned by the primary network provider (whose users are referred to as primary users) using spectrum underlay or spectrum overlay. Spectrum underlay and overlay techniques are the basis for designing the emerging cognitive radio networks. Also, in contrast to resource allocation problems in traditional wireless networks [4]-[6], in cognitive radio networks primary users should be protected while secondary users access the spectrum.

In the spectrum overlay paradigm, secondary users are only allowed to access spectrum resources (i.e., channels) owned by the primary network provider if these channels are not being used by primary users. Here, spectrum sensing needs to be performed to avoid possible collision with primary users [7], [8]. Given that the spectrum opportunities are detected by a spectrum sensing technique, it is important to coordinate the spectrum sharing among secondary users in such a way that different design objectives can be achieved. Centralized architectures for spectrum sensing and sharing in cognitive wireless networks were proposed in [9] and [10]. In general, a medium access control (MAC) layer protocol with sensing capability is important to perform fair resource allocation among secondary users while avoiding collision with primary users.

For the spectrum underlay paradigm, it is required that an interference limit corresponding to an interference temperature level be maintained at receiving points of the primary network. A graph-theoretic model for spectrum sharing/access among secondary users was proposed in [11] where different objective functions were investigated. In [12], the channel allocation problem was formulated using game theory. Here, the proposed utility functions capture the interference perceived by one user on each channel and/or the interference this user creates to its neighboring users. In these papers, primary users were not explicitly protected from interference due to spectrum access by secondary users. In [13], a heuristicbased channel and power allocation algorithm was proposed where interference constraints for primary users were considered. However, how good the performance of the proposed algorithm compared to the optimal solution was not known. In [14], a user removal algorithm based on the treepruning algorithm was proposed so that QoS constraints for secondary users and interference temperature constraints for primary users are satisfied. The proposed removal algorithm is, however, computationally extensive.

In this paper, we propose a resource allocation frame- 
work for spectrum underlay in cognitive wireless networks. Specifically, we consider design problems of cognitive wireless networks using code-division multiple access (CDMA) technology with explicit interference protection for primary users and QoS constraints for secondary users. We assume that secondary users have the requirement of a minimum transmission rate in terms of SINR (or equivalent bit error rate (BER)) and maximum power constraints. When the network load is high, we show how to perform admission control to guarantee QoS constraints for secondary users and interference constraints for primary users. If all of the secondary users can be supported, we solve the joint rate and power allocation problems under two popular fairness criteria, namely, the maxmin and the proportional fairness (PF) criteria [15], [16].

Similar design problems exist in conventional wireless networks. In [17], different user removal algorithms for joint admission control and power control were proposed which employed the power control technique developed in [18]. These admission control algorithms were proposed for the traditional cellular wireless networks and did not take into consideration the interference constraints which are present in a cognitive radio network based on spectrum underlay. Moreover, joint rate and power allocation problems in CDMA wireless network were solved in several works in the literature [19]-[21]. Most of the existing works in the literature, however, aim to maximize the network throughput. However, it is important to maintain throughput fairness among users to avoid severe QoS degradation for users with unfavorable channel conditions. In addition, for the spectrum sharing problem, both QoS and interference constraints need to be considered. These design aspects will be considered in the resource allocation framework proposed in this paper.

The rest of this paper is organized as follows. System model and problem definition are presented in Section II. Section III presents the solutions for the admission control problem. The solutions of the power/rate allocation problem for the spectrum underlay model are described in Section IV. Section V presents the numerical results. Conclusions are stated in Section VI.

\section{System Model ANd Problem Definition}

We consider the hierarchical spectrum sharing problem in a cognitive wireless network where several unlicensed users (secondary users) and licensed users (primary users) transmit data in the same spectral region. Specifically, the spectrum underlay model is investigated in this paper where secondary users and primary users transmit simultaneously in a common frequency band (e.g., as in a CDMA-based wireless network). Interested readers are referred to [3] for an excellent survey and for more details about the taxonomy of these spectrum access models. The entities we work with are the communication links each of which corresponds to a pair of users who wish to communicate with each other. We will refer to the communication links belonging to secondary networks as secondary links.

We assume that there are a number of primary and secondary users communicating with their partners simultaneously. Here, the term "user" will be used broadly where it can be a mobile node or base station/access point in a centralized network or simply a mobile node in an ad hoc network. The CDMA technology will be assumed although the model may be extended for other technologies as well.

Simultaneous communications among users (i.e., both primary and secondary users) will interfere with each other. We will consider the interference constraints at the receiving ends of the primary network which will be referred to as primary receiving points in the sequel. We assume that each primary receiving point can tolerate a maximum interference level. Also, secondary links have desired QoS performance in terms of BER. Fig. 1 illustrates the transmission setting considered in this paper.

\section{A. Modeling of QoS and Interference Constraint}

Assume that there are $M$ primary receiving points and $N$ secondary communication links in the considered geographical area. Let us denote the channel gain from the transmitting node of secondary link $i$ to receiving node of secondary link $j$ by $g_{j, i}^{(s)}$ while the channel gain from the transmitting node of secondary link $i$ to primary receiving point $j$ as $g_{j, i}^{(p)}$. If $N_{i}$ denotes the total noise and interference due to primary users' transmissions at the receiving side of secondary link $i$, for wireless access based on CDMA, the corresponding effective bit-energy-to-noise power spectral density ratio can be written as [19]

$$
\mu_{i}=\frac{B}{R_{i}} \frac{g_{i, i}^{(s)} P_{i}}{\sum_{j=1, j \neq i}^{N} g_{i, j}^{(s)} P_{j}+N_{i}}
$$

where $B$ is the spectrum bandwidth, $R_{i}$ is the transmission rate of secondary link $i$. Here, $B / R_{i}$ is the processing gain which is usually required to be larger than a particular value. The processing gain is simply equal to one for other multiple access technologies such as FDMA and $\mu_{i}$ denotes the SINR. In the sequel, we will refer to $\mu_{i}$ as SINR in all cases. Now, if a particular modulation scheme is employed, there will be an explicit relationship between BER and SINR. Thus, for a specific required level of BER for secondary link $i, \mu_{i}$ is required to be larger than a corresponding value $\gamma_{i}$. Hence, the QoS requirement for secondary link $i$ can be expressed as

$$
\mu_{i} \geq \gamma_{i}, \quad i=1,2, \cdots, N
$$

Now, let $I_{j}$ be the maximum interference level tolerable by primary receiving point $j$. The interference constraint for primary receiving point $j$ can be written as

$$
\sum_{i=1}^{N} g_{j, i}^{(p)} P_{i} \leq I_{j}, \quad j=1,2, \cdots, M
$$

where total interference at the primary receiving point $j$ should be smaller than the tolerable limit. We assume that transmission rate of secondary link $i$ can be adjusted in an allowable range where the minimum and the maximum values are $R_{i}^{\min }$ and $R_{i}^{\max }$, respectively. Also, power of secondary link $i$ is constrained to be smaller than the maximum limit $P_{i}^{\max }$. We assume that all channel gains can be made available at a control point. 
Remark: If the channel is frequency-selective and an L-finger $R A K E$ receiver is used at each receiver, the effective bitenergy-to-noise spectral density ratio can be written as [21]

$$
\mu_{i}=\frac{B}{R_{i}} \frac{P_{i} \sum_{l=1}^{L} g_{i, i, l}^{(s)}}{\frac{2}{3} \sum_{j=1, j \neq i}^{N} P_{j} \sum_{l=1}^{L} g_{i, j, l}^{(s)}+N_{i}}
$$

where $g_{i, j, l}^{(s)}$ is the channel gain for the l-th path from the transmitter of link $j$ to the receiver of link $i$. Let us denote $g_{i, i}^{(s)}=\sum_{l=1}^{L} g_{i, i, l}^{(s)}$ and $g_{i, j}^{(s)}=2 / 3 \sum_{l=1}^{L} g_{i, j, l}^{(s)}(i \neq j)$. Substituting these quantities into (4), we can obtain equation (1) with the corresponding "equivalent" channel gains $g_{i, j}^{(s)}$. As a consequence, the same analysis can be applied to both cases.

\section{B. Admission Control Problem}

The admission control problem considered in this paper is similar to that investigated in [17]. Specifically, secondary links requesting access to the spectrum licensed to the primary network have QoS requirements expressed in (2). The major challenge for the spectrum sharing problem comes from the interference protection for primary users expressed in (3) which is usually not considered in the conventional fixed spectrum allocation paradigm.

In particular, we are interested in the scenario where a number of secondary links wish to access the spectrum with minimum transmission rate (i.e., $R_{i}=R_{i}^{\mathrm{min}}$ ) and both the QoS requirements (in (2)) as well as the interference constraints (in (3)) are satisfied. The problem is how to choose the subset of requesting links with maximum size such that the constraints in (2) and (3) are both satisfied.

\section{Joint Rate and Power Allocation Problem}

When the network load is low, all requesting secondary links with minimum transmission rates can be supported while satisfying the QoS and the interference constraints in (2) and (3), respectively. If this is the case, secondary links would increase their transmission rates above the minimum values and share the spectrum in a fair manner. For notational convenience, we will arrange power, rate and other quantities of all secondary links into the corresponding vectors. For example, $P$ will denote a column vector whose element $P_{i}$ is the transmission power of secondary link $i$. The joint rate and power allocation problem can be stated as

$$
\begin{array}{ll}
\operatorname{maximize} & f(R) \\
\text { subject to } & R^{\min } \preceq R \preceq R^{\max }, P \preceq P^{\max } \\
& \text { and constraints }(2),(3)
\end{array}
$$

where the objective function $f(R)$ strikes a balance between maximizing throughput and maintaining fairness for different secondary links. Specifically, we will investigate two fairness criteria, i.e., $f(R)=\max \left\{\min _{i} R_{i}\right\}$ to achieve max-min fairness and $f(R)=\sum_{i=1}^{N} \ln \left(R_{i}\right)$ to achieve proportional fairness [15], [16]. We will show how to solve the admission control as well as the joint rate and power allocation problems in Sections III and IV, respectively.

\section{Admission CONTROL Algorithms}

As has been mentioned in Section II, we will consider the admission control problem when the network load is high and all of the secondary links transmit with their minimum rate (if admitted). Now, using equation (1), we can rewrite the QoS constraint in (2) as follows:

$P_{i} \geq \sum_{j=1, j \neq i}^{N} \frac{\gamma_{i} R_{i}^{\min }}{B} \frac{g_{i, j}^{(s)}}{g_{i, i}^{(s)}} P_{j}+\frac{\gamma_{i} R_{i}^{\min }}{B} \frac{N_{i}}{g_{i, i}^{(s)}}, \quad i=1,2, \cdots, N$.

These constraints for all secondary links can be written in the matrix form as follows:

$$
(I-F) P \succeq u
$$

where $I$ is an identity matrix of order $N \times N, u$ is a column vector which can be written as

$u=\left(\frac{\gamma_{1} R_{1}^{\min }}{B} \frac{N_{1}}{g_{1,1}^{(s)}}, \frac{\gamma_{2} R_{2}^{\min }}{B} \frac{N_{2}}{g_{2,2}^{(s)}}, \cdots, \frac{\gamma_{N} R_{N}^{\min }}{B} \frac{N_{N}}{g_{N, N}^{(s)}}\right)^{\prime}$

where $(.)^{\prime}$ denotes the matrix/vector transpose. And $F$ is an $N \times N$ matrix whose $(i, j)$-th element is

$$
F_{i, j}= \begin{cases}\frac{\gamma_{i} R_{i}^{\min }}{B} \frac{g_{i, j}^{(s)}}{g_{i, i}^{(s)}}, & \text { if } i \neq j \\ 0, & \text { if } i=j .\end{cases}
$$

\section{A. Constrained Power Control}

Recall that we are interested in a scenario where not all $N$ secondary links can be admitted into the network while satisfying the QoS and the interference constraints stated in (2) and (3), respectively. We will first focus on the power allocation problem under maximum power constraint (i.e., $P \preceq P^{\max }$ ) and QoS constraints while ignoring the interference constraints for the time being.

In [18], the authors proposed an efficient iterative power control algorithm which can be implemented synchronously or asynchronously. Specifically, let $P_{i}(t)$ and $P_{i}(t+\Delta t)$ denote the power levels of secondary link $i$ after two consecutive power updates at time instants $t$ and $t+\Delta t$, respectively. The power of secondary link $i$ at time $t+\Delta t$ is updated as follows:

$$
P_{i}(t+\Delta t)=\min \left\{P_{i}^{\max }, P_{i}(t) \frac{\gamma_{i}}{\mu_{i}(t)}\right\}
$$

where $\mu_{i}(t)$ is the instantaneous SINR at the receiving side of secondary link $i$ at time instant $t$ with power vector $P(t)$. It was shown in [18] that this power control algorithm converges to the fixed point solution of

$$
P=\min \left\{P^{\max }, F P+u\right\}
$$

which will be referred to as the stationary power vector. Let $\Omega$ be the set of secondary links and $P^{\Omega}$ be the stationary power vector when the power algorithm with the rule as in (8) is run with the secondary link set $\Omega$. From the results obtained in [17], we have the following facts:

Fact 1: If all secondary links in $\Omega$ can be supported (i.e., the power control algorithm in (8) results in a stationary power vector $P^{\Omega}$ satisfying the QoS constraints in (2)), the QoS constraints will be satisfied with equality. 
Fact 2: If a subset $\Omega^{\prime} \subseteq \Omega$ is the set of secondary links which are not supported with stationary power vector $P^{\Omega}$, then $P_{i}^{\Omega}=P_{i}^{\max }$ for $i \in \Omega^{\prime}$.

Now, let us define the following "interference measures":

$$
\begin{aligned}
\alpha_{i}\left(P^{\Omega}\right) & =\left[P_{i}^{\Omega} \sum_{j=1, j \neq i}^{N} g_{j, i}^{(s)}+N_{i}\right]-\frac{g_{i, i}^{(s)}}{\gamma_{i}} \frac{B}{R_{i}} P_{i}^{\Omega(10)} \\
\beta_{i}\left(P^{\Omega}\right) & =\left[\sum_{j=1, j \neq i}^{N} g_{i, j}^{(s)} P_{j}^{\Omega}+N_{i}\right]-\frac{g_{i, i}^{(s)}}{\gamma_{i}} \frac{B}{R_{i}} P_{i}^{\Omega(11)} \\
D^{\Omega_{0}}\left(P^{\Omega}\right) & =\sum_{i \in \Omega_{0}} \beta_{i}\left(P^{\Omega}\right), \quad \text { for } \Omega_{0} \subseteq \Omega .
\end{aligned}
$$

We can easily see that

$$
D^{\Omega}\left(P^{\Omega}\right)=\sum_{i \in \Omega} \beta_{i}\left(P^{\Omega}\right)=\sum_{i \in \Omega} \alpha_{i}\left(P^{\Omega}\right)
$$

We can also see that if the QoS constraint for secondary link $i$ is satisfied with equality, then $\beta_{i}\left(P^{\Omega}\right)=0$. Also, $D^{\Omega}\left(P^{\Omega}\right)=0$ if and only if all secondary links in $\Omega$ are supported. In general, we have $\beta_{i}\left(P^{\Omega}\right) \geq 0$ and the value of $\beta_{i}\left(P^{\Omega}\right)$ reflects the degree by which the QoS constraint for secondary link $i$ is violated. Also, it is intuitive that $\alpha_{i}\left(P^{\Omega}\right)$ quantifies the aggregate relative interference that secondary link $i$ creates for other links in $\Omega$. We will present single and multiple link removal algorithms for admission control in the following subsection.

\section{B. Admission Control with QoS and Interference Constraints}

In [17], the authors proposed several removal algorithms which aim at maximizing the number of secondary links which can be admitted into the network while satisfying the QoS requirements. Among these proposed algorithms, SMIRA and SMART(R) are the two most efficient ones. Note that the interference measures defined in (10)-(12) are not the same as those in [17]. However, the spirit of the SMIRA and SMART(R) algorithms remain the same in this paper.

1) Single Removal Algorithm: SMIRA runs the power control algorithm in (8) until reaching the stationary power vector. Based on this stationary power vector, the algorithm removes one link from the network and runs the power control algorithm again. This procedure is repeated until the remaining set of links can be supported. The removal criterion of SMIRA is as follows:

$$
i^{*}=\operatorname{argmax}_{i \in \Omega}\left\{\max \left(\alpha_{i}\left(P^{\Omega}\right), \beta_{i}\left(P^{\Omega}\right)\right)\right\} .
$$

Intuitively, SMIRA algorithm removes the link which violates the QoS constraints the most and/or creates the largest amount of interference to other links in each removal step. Thus, it can potentially remove the least number of links from the network. In our spectrum sharing problem, besides QoS constraint, admission and power control should be done such that interference constraints for primary links stated in (3) are also satisfied. We have the following result on the complexity of this admission control problem.

Proposition 1: The admission control problem with QoS and interference constraints is NP-hard.
Proof: The proof is provided in Appendix A.

Because of the complexity of the problem, we propose a low-complexity admission control algorithm in this subsection. The proposed algorithm also removes the "worst" link oneby-one. In each step, we perform the power control algorithm as in (8) and remove one secondary link from the network until the remaining set of links satisfies both the QoS and the interference constraints. Here, the key issue is to construct a removal criterion which achieves good overall performance. Because there are two different kinds of constraints, we consider the following cases in each removal step.

Case 1: Interference constraints for all primary receiving points stated in (3) are satisfied but QoS constraints in (2) are violated.

In this case, we employ the SMIRA algorithm as presented in Section III.A.

Case 2: Interference constraints for primary receiving points stated in (3) are violated.

Note that this case covers both the scenarios where QoS constraints in (2) are violated or not. In this case, we would remove the link which violates both QoS and interference constraints the most in each step. Now, we define the measure which quantifies degree of violation at primary receiving point $j$ as follows:

$$
\eta_{j}(\Omega)=I_{j}-\sum_{i=1}^{N} g_{j, i}^{(p)} P_{i}^{\Omega} .
$$

We propose the following removal criterion in which the removal metric quantifies the aggregate interference effect that each link creates to other links:

$$
\begin{aligned}
i^{*} & \underset{i \in \Omega}{\operatorname{argmax}}\left\{\frac{D^{\Omega}\left(P^{\Omega}\right)}{D^{\Omega}\left(P^{\Omega}\right)+\sum_{k=1}^{M} \eta_{k}(\Omega)} \times\right. \\
& \max \left[\sum_{j \in \Omega, j \neq i} g_{j, i}^{(s)} P_{i}^{\Omega}, \sum_{j \in \Omega, j \neq i} g_{i, j}^{(s)} P_{j}^{\Omega}\right] \\
& \left.+\sum_{j=1}^{M} \frac{\eta_{j}(\Omega)}{D^{\Omega}\left(P^{\Omega}\right)+\sum_{k=1}^{M} \eta_{k}(\Omega)} g_{j, i}^{(p)} P_{i}^{\Omega}\right\} .
\end{aligned}
$$

In fact, $\sum_{j \in \Omega, j \neq i} g_{j, i}^{(s)} P_{i}^{\Omega}$ denotes the total interference that secondary link $i$ creates to other secondary links while $\sum_{j \in \Omega, j \neq i} g_{i, j}^{(s)} P_{j}^{\Omega}$ is the total interference received at the receiving end of link $i$. Moreover, $g_{j, i}^{(p)} P_{i}^{\Omega}$ denotes the interference that secondary link $i$ creates for primary receiving point $j$. Recall that $D^{\Omega}\left(P^{\Omega}\right)$ quantifies the degree of violation for QoS constraints and $\eta_{j}(\Omega)$ quantifies the degree of violation for the interference constraint of primary receiving point $j$. Therefore, the proposed criterion removes in each step the secondary link which creates the largest amount of interference for primary receiving points and receives or creates the largest amount of interference from/for other secondary links in the weighted average sense. As a result, it would potentially remove the least number of secondary links from the network. We will refer to this algorithm as interference constraintaware SMIRA (I-SMIRA) in the sequel. The computational complexity of I-SMIRA is only of $O\left(N^{2}\right)$. 
2) Multiple Removal Algorithm: In practice, it may be desired to have a fast admission control algorithm, especially when many links are to be removed in a highly congested network. In this case, multiple links may be removed from the network in each removal step. In [17], the authors proposed the SMART(R) algorithm which removes $R$ links from the network in each step. Before presenting the removal metric, let us define

$$
P_{i}^{\Omega / \Omega_{0}}= \begin{cases}P_{i}^{\Omega}, & \text { if } i \in \Omega_{0} \\ 0, & \text { otherwise. }\end{cases}
$$

Now, the SMART(R) algorithm removes links in the set $\Omega \backslash \Omega_{0}$ which attains

$$
\max _{\Omega_{0} \subset \Omega}\left\{D^{\Omega}\left(P^{\Omega}\right)-D^{\Omega_{0}}\left(P^{\Omega / \Omega_{0}}\right)\right\}
$$

where $\left|\Omega \backslash \Omega_{0}\right|=R$ and recall that $D^{\Omega_{0}}\left(P^{\Omega / \Omega_{0}}\right)=$ $\sum_{i \in \Omega_{0}} \beta_{i}\left(P^{\Omega / \Omega_{0}}\right)$. In essence, the SMART(R) algorithm removes a set of links of size $R$ which contributes the most to $D^{\Omega}\left(P^{\Omega}\right)$. Note that $D^{\Omega}\left(P^{\Omega}\right)$ reflects the degree of violation of QoS constraints. Thus, the SMART(R) algorithm tends to remove the set of worst links in each removal step. To simplify the calculation of (18), we can use the following result.

Proposition 2: The removal metric of the SMART(R) algorithm can be rewritten as

$$
\begin{aligned}
& D^{\Omega}\left(P^{\Omega}\right)-D^{\Omega_{0}}\left(P^{\Omega / \Omega_{0}}\right)=\sum_{i \notin \Omega_{0}}\left[\beta_{i}\left(P^{\Omega}\right)+\alpha_{i}\left(P^{\Omega}\right)\right. \\
& \left.+\left(\frac{g_{i, i}^{(s)}}{\gamma_{i}} \frac{B}{R_{i}} P_{i}^{\Omega}-N_{i}\right)\right]-\left[\sum_{i \notin \Omega_{0}} \sum_{j \notin \Omega_{0}, j \neq i} g_{i, j}^{(s)} P_{j}^{\Omega}\right] .
\end{aligned}
$$

Proof: The proof is provided in Appendix B.

The formula to calculate the removal metric in this proposition is simpler to compute because it only calculates all the terms over the removal set of size $R$ which is usually very small. Note that if $R=1$, the SMART(R) algorithm removes a single link in each removal step. Now, for our admission control problem with both QoS and interference constraints, we propose an interference constraint-aware SMART(R) removal algorithm which will be referred to as I-SMART(R) in the sequel. Similar to the I-SMIRA algorithm, we consider the following cases in each removal step.

Case 1: Interference constraints for all primary receiving points stated in (3) are satisfied but QoS constraints in (2) are violated.

In this case, we employ the $\operatorname{SMART(R)}$ algorithm as presented above.

Case 2: Interference constraints for primary receiving points stated in (3) are violated

In this case, we remove the set of links $\Omega_{1}=\Omega \backslash \Omega_{0}$ of size
$R$ in each removal step which satisfies

$$
\begin{aligned}
& \Omega_{1}=\underset{\Omega_{1}}{\operatorname{argmax}}\left\{\frac{D^{\Omega}\left(P^{\Omega}\right)}{D^{\Omega}\left(P^{\Omega}\right)+\sum_{k=1}^{M} \eta_{k}(\Omega)}\right. \\
& \times \sum_{i \in \Omega_{1}} \max \left\{\sum_{j \in \Omega, j \neq i} g_{j, i}^{(s)} P_{i}^{\Omega}, \sum_{j \in \Omega, j \neq i} g_{i, j}^{(s)} P_{j}^{\Omega}\right\} \\
& \left.\quad+\sum_{i \in \Omega_{1}} \sum_{j=1}^{M} \frac{\eta_{j}(\Omega)}{D^{\Omega}\left(P^{\Omega}\right)+\sum_{k=1}^{M} \eta_{k}(\Omega)} g_{j, i}^{(p)} P_{i}^{\Omega}\right\} .
\end{aligned}
$$

In fact, the removal metric in (20) is similar to that for single removal in (16). However, the total interference effects of the potential removal set is accounted for in this metric. Note that $\Omega_{0}$ is the remaining set of links after $R$ links in the set $\Omega_{1}=$ $\Omega \backslash \Omega_{0}$ have been removed from the network. As before, the power control algorithm in (8) is run until the stationary power vector is attained before the removal algorithm is activated. The computational complexity of I-SMART(R) is of $O\left(N^{2}\right)$ which is the same as that of I-SMIRA algorithm.

\section{Some Implementation Issues}

In order to guarantee both the QoS requirements for secondary users and interference constraints for primary users, all channel gains (i.e., $g_{i, j}^{(s)}$ and $g_{i, j}^{(p)}$ ) must be continuously estimated for online admission control operation. Also, performing distributed power and admission control for spectrum underlay may be difficult because of the following reasons. First, interference constraints are required to be satisfied at all times in general. This requirement may only be fulfilled by centralized implementation because secondary links are not allowed to cause even a short-term violation of QoS and interference constraints as in the conventional wireless networks [17], [18]. Second, primary users usually do not assist secondary users in estimating the channel gains $g_{i, j}^{(p)}$. Thus, secondary users are responsible for making these channel gains available at the central controller for power and admission control purposes.

The channel gains between secondary links (i.e., $g_{i, j}^{(s)}$ ) can be estimated by using pilot-aided or other approaches [22], [23]. Channel gains from transmitters of secondary links to primary receiving points (i.e., $g_{i, j}^{(p)}$ ) can be estimated by employing sensors near all receiving points which are then forwarded to the central controller [8].

\section{Optimization of Joint Rate AND POWER ALLOCATION}

When the network load is low, all secondary links can be admitted into the network. In this case, they can increase their transmission rates above the minimum values. In this section, we show how to solve the optimization problem stated in (5). The decision variables are transmission rates $R$ and powers $P$. As in [19], we will assume that continuous rate and rate variation is achieved by varying the processing gain. The results obtained in this paper, therefore, can be considered as upper bounds for the discrete case. 


\section{A. Rate and Power Allocation with Max-Min Fairness}

It is observed that the objective function in (5) is equivalent to $\min \left\{\max _{i} 1 / R_{i}\right\}$. By introducing a new variable $t$ and writing down all the constraints explicitly, the optimization problem (5) becomes equivalent to

$$
\begin{aligned}
& \text { minimize } \quad t \\
& \text { subject to } \\
& 1 / R_{i} \leq t, \quad i=1,2, \cdots, N \\
& \frac{B}{R_{i}} \frac{g_{i, i}^{(s)} P_{i}}{\sum_{j=1, j \neq i}^{N} g_{i, j}^{(s)} P_{j}+N_{i}} \geq \gamma_{i}, \quad i=1,2, \cdots, N \\
& \sum_{i=1}^{N} g_{j, i}^{(p)} P_{i} \leq I_{j}, \quad j=1,2, \cdots, M \\
& R_{i}^{\min } \leq R_{i} \leq R_{i}^{\max }, P_{i} \leq P_{i}^{\max }, \quad i=1,2, \cdots, N .
\end{aligned}
$$

This optimization problem is not convex. However, we can transform it into a geometric program which can be solved efficiently (Chapter 4, [25]). Specifically, the optimization problem in (21) is equivalent to

$$
\begin{aligned}
& \text { minimize } t \\
& \text { subject to } \\
& t^{-1} R_{i}^{-1} \leq 1, \quad i=1,2, \cdots, N \\
& \frac{\gamma_{i}}{B g_{i, i}^{(s)}} R_{i} P_{i}^{-1} \sum_{j=1, j \neq i}^{N} g_{i, j}^{(s)} P_{j}+\frac{\gamma_{i} N_{i}}{B g_{i, i}^{(s)}} R_{i} P_{i}^{-1} \leq 1 \\
& \quad i=1,2, \cdots, N \\
& \sum_{i=1}^{N} \frac{g_{j, i}^{(p)}}{I_{j}} P_{i} \leq 1, \quad j=1,2, \cdots, M \\
& R_{i}^{\min } R_{i}^{-1} \leq 1,\left(R_{i}^{\max }\right)^{-1} R_{i} \leq 1,\left(P_{i}^{\max }\right)^{-1} P_{i} \leq 1 \\
& \quad i=1,2, \cdots, N
\end{aligned}
$$

Now, let us define $P_{i}=e^{x_{i}}, R_{i}=e^{y_{i}}$ and $t=e^{s}$, substitute these new variables into (22), and take $\ln$ in both the objective and the constraint functions. Then, we have the following optimization problem:

$$
\begin{aligned}
& \text { minimize } s \\
& \text { subject to } \\
& \ln \left(e^{\left(-s-y_{i}\right)}\right) \leq 0, \quad i=1,2, \cdots, N \\
& \ln \left(\frac{\gamma_{i}}{B g_{i, i}^{(s)}} \sum_{j=1, j \neq i}^{N} g_{i, j}^{(s)} e^{\left(x_{j}-x_{i}+y_{i}\right)}+\frac{\gamma_{i} N_{i}}{B g_{i, i}^{(s)}} e^{\left(y_{i}-x_{i}\right)}\right) \leq 0, \\
& \ln \left(\sum_{i=1}^{N} \frac{g_{j, i}^{(p)}}{I_{j}} e^{x_{i}}\right) \leq 0, \quad j=1,2, \cdots, M \\
& \ln \left(R_{i}^{\min } e^{-y_{i}}\right) \leq 0, \ln \left(\left(R_{i}^{\max }\right)^{-1} e^{y_{i}}\right) \leq 0, \\
& \ln \left(\left(P_{i}^{\max }\right)^{-1} e^{x_{i}}\right) \leq 0, \quad i=1,2, \cdots, N \text {. }
\end{aligned}
$$

Since log-sum-exp functions are convex [25], this optimization problem is a convex one. Therefore, the globally optimal solution can be found by interior point methods [25]. We have the following property on the solution of the joint rate and power allocation problem.
Proposition 3: The optimal solution of the joint rate and power allocation problem satisfies $R_{i}=R_{j}, \forall i, j$.

Proof: The proof is provided in Appendix C.

Hence, the rate and power allocation problem under maxmin fairness achieves perfectly fair rates for all secondary links in the sense that optimal transmission rates for all links are the same.

\section{B. Rate and Power Allocation with Proportional Fairness}

For proportional fairness criterion, the objective function $f(R)=\sum_{i=1}^{N} \ln \left(R_{i}\right)$ can be rewritten as

$$
\operatorname{maximize} \ln \left(\prod_{i=1}^{N} R_{i}\right) \text {. }
$$

This objective function is equivalent to $\min \left(1 / \prod_{i=1}^{N} R_{i}\right)$. After changing variables and using the procedure as in Section IV. A, the optimization in (5) with proportional fairness criterion can be written as

$$
\begin{aligned}
& \text { minimize }-\sum_{i=1}^{N} y_{i} \\
& \text { subject to } \\
& \ln \left(\frac{\gamma_{i}}{B g_{i, i}^{(s)}} \sum_{j=1, j \neq i}^{N} g_{i, j}^{(s)} e^{\left(x_{j}-x_{i}+y_{i}\right)}+\frac{\gamma_{i} N_{i}}{B g_{i, i}^{(s)}} e^{\left(y_{i}-x_{i}\right)}\right) \leq 0, \\
& \ln \left(\sum_{i=1}^{N} \frac{g_{j, i}^{(p)}}{I_{j}} e^{x_{i}}\right) \leq 0, \quad j=1,2, \cdots, N \\
& \ln \left(R_{i}^{\min } e^{-y_{i}}\right) \leq 0 \ln \left(\left(R_{i}^{\max }\right)^{-1} e^{y_{i}}\right) \leq 0, \\
& \ln \left(\left(P_{i}^{\max }\right)^{-1} e^{x_{i}}\right) \leq 0, \quad i=1,2, \cdots, N .
\end{aligned}
$$

This equivalent optimization problem is convex. Therefore, the globally optimal solution can be obtained using standard convex optimization techniques.

\section{Numerical RESUltS}

We consider a simple network setting as shown in Fig. 1. Assume that primary users communicate with its BS in the uplink direction (i.e., a single cell is considered). Transmitting nodes corresponding to the secondary links are randomly located in a rectangular area and the BS of primary network is located at the center of the rectangular area. The size of the rectangular area is $2000 \mathrm{~m} \times 2000 \mathrm{~m}$. Also, receiving node of each secondary link is generated randomly in a $1000 \mathrm{~m} \times$ $1000 \mathrm{~m}$ rectangle with its transmitting node being at the center.

The channel gains are modeled as $g_{i, j}^{(s)}=$ $K_{0} \cdot 10^{\beta_{i, j}^{(s)} / 10} \cdot\left(d_{i, j}^{(s)}\right)^{-4}, g_{i, j}^{(p)}=K_{0} \cdot 10^{\beta_{i, j}^{(p)} / 10} \cdot\left(d_{i, j}^{(p)}\right)^{-4}$, where $d_{i, j}^{(s)}$ and $d_{i, j}^{(p)}$ are the corresponding distances, $\beta_{i, j}^{(s)}$ and $\beta_{i, j}^{(p)}$ are random Gaussian variables with zero mean and standard deviation equal $6 \mathrm{~dB}, K_{0}=10^{3}$ which captures system and transmission effects such as antenna gain, carrier frequency, etc. The total noise and interference at the receiving node of all secondary links is chosen to be $N_{i}=N_{0}=10^{-10}$ W. The maximum transmission power on secondary links is $P_{i}^{\max }=0.1 \mathrm{~W}$. The spectrum bandwidth is $B=5.12$ $\mathrm{MHz}$. We will denote the tolerable interference limit at 


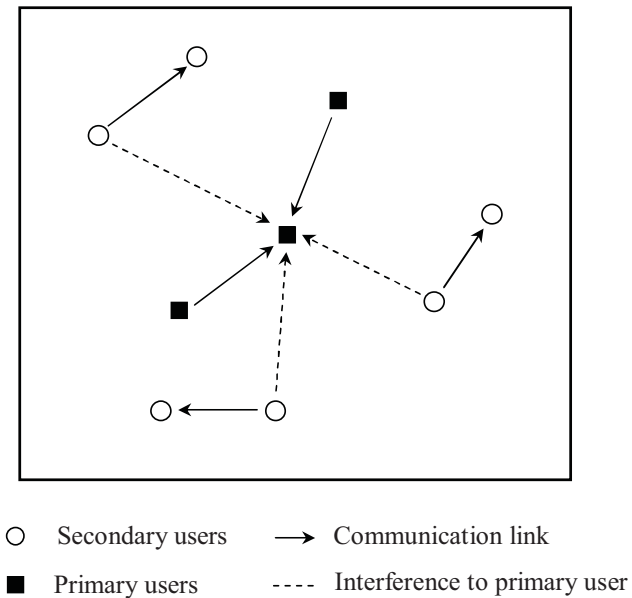

Fig. 1. Spectrum sharing among primary and secondary users.

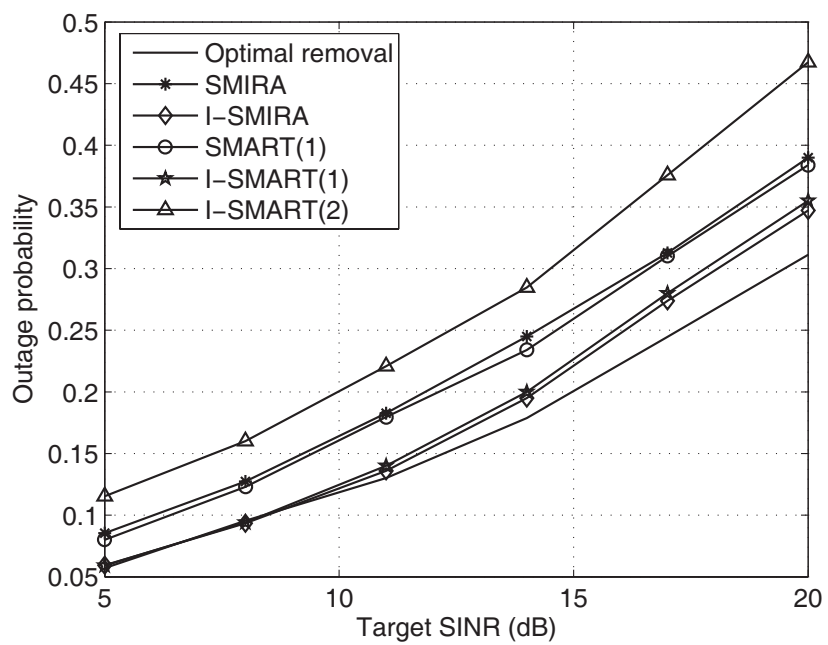

Fig. 2. Outage probability versus target SINR of different removal algorithms (for $I=5 N_{0}, N=7$ ).

the primary receiving point (i.e., BS) as $I$. The minimum transmission rate on secondary links is $R_{i}^{\min }=64 \mathrm{kbps}$ and the maximum transmission rate is $R_{i}^{\max }=B / P G$, where $P G$ is the minimum processing gain. For each simulation run, the locations of secondary links (i.e., transmitting and receiving nodes) are generated randomly. The measure of interest is obtained by averaging over $10^{3}$ simulation runs.

We will investigate performance of four removal algorithms, namely, SMIRA, SMART(R), I-SMIRA, and I-SMART(R) algorithms. For SMIRA and SMART(R) algorithms, we remove links in each removal step using the metrics in (14), (18), respectively, until the remaining links satisfy both the QoS and the interference constraints. In contrast, we consider two different cases in each removal step as presented in Section III.B for the I-SMIRA, I-SMART(R) removal algorithms. Two performance measures will be investigated, namely, outage probability and number of admitted links. Outage probability is calculated as the ratio of average number of removed links and average number of requesting links.

The outage probability versus target SINR under different set of constraints for different removal algorithms and optimal removal is shown in Figs. 2-3. Results for optimal removal

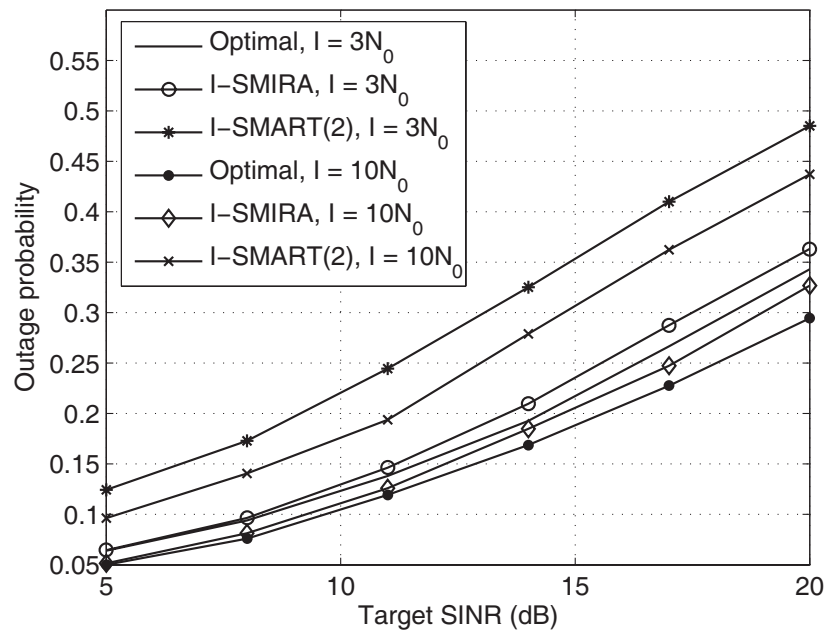

Fig. 3. Outage probability versus target SINR for different interference constraint requirements (for $N=7$ ).

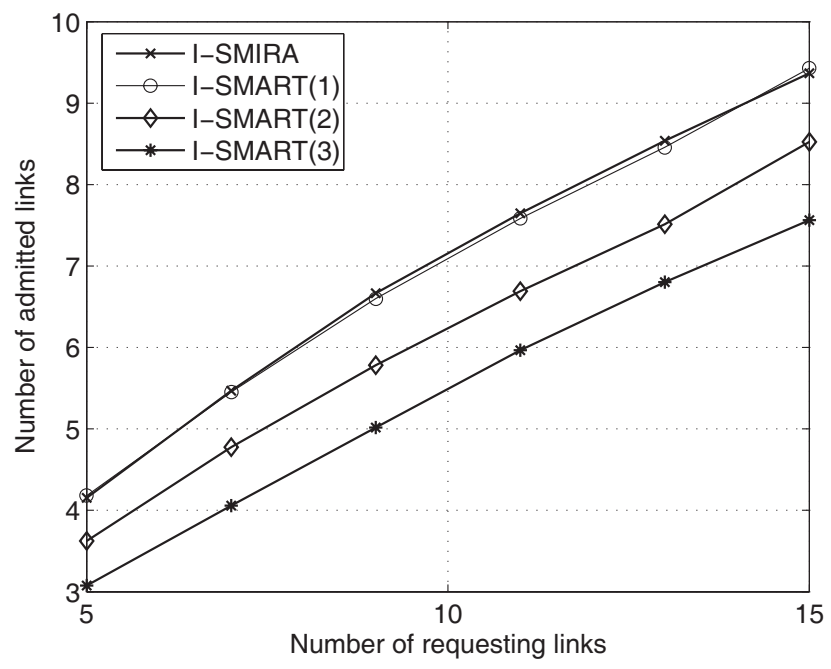

Fig. 4. Number of admitted links versus number of requesting links from secondary users (for $I=5 N_{0}, \operatorname{SINR}=15 \mathrm{~dB}$ ).

are obtained by exhaustive search. As is evident, both of the proposed single removal algorithms (i.e., I-SMIRA, ISMART(1)) achieve outage probability very close to that of optimal removal, especially for low and medium values of target SINR (i.e., target SINR $<10 \mathrm{~dB}$ ). Fig. 2 shows that SMIRA and SMART(1) algorithms, which do not account for interference constraints in the removal metrics, consistently achieve higher outage probability than I-SMIRA and I-SMART(1) for all target SINR values. Outage performance for I-SMART(2), which removes two links in each removal step, is also shown in these two figures. It can be observed that I-SMART(2) has higher outage probability than all other removal algorithms.

We illustrate the number of admitted links and outage probability versus the number of requesting links for different removal algorithms in Figs. 4-5, respectively. These two figures show that I-SMIRA and I-SMART(1) algorithms achieve almost the same outage performance. In addition, outage performance of I-SMART(R) algorithm degrades rapidly with the number of removed links $R$. In order to quantify the tradeoff 


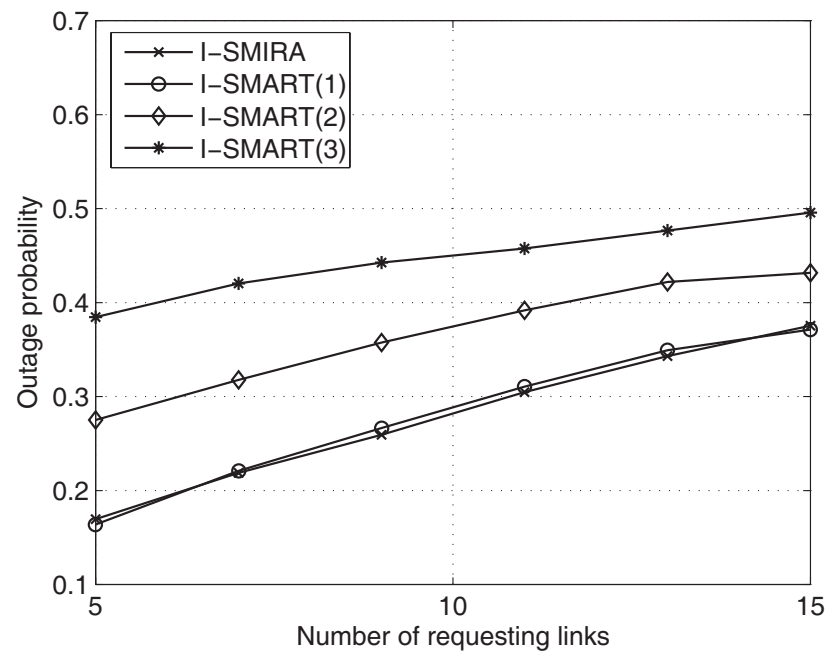

Fig. 5. Outage probability versus number of requesting links from secondary users (for $I=5 N_{0}$, SINR $=15 \mathrm{~dB}$ ).

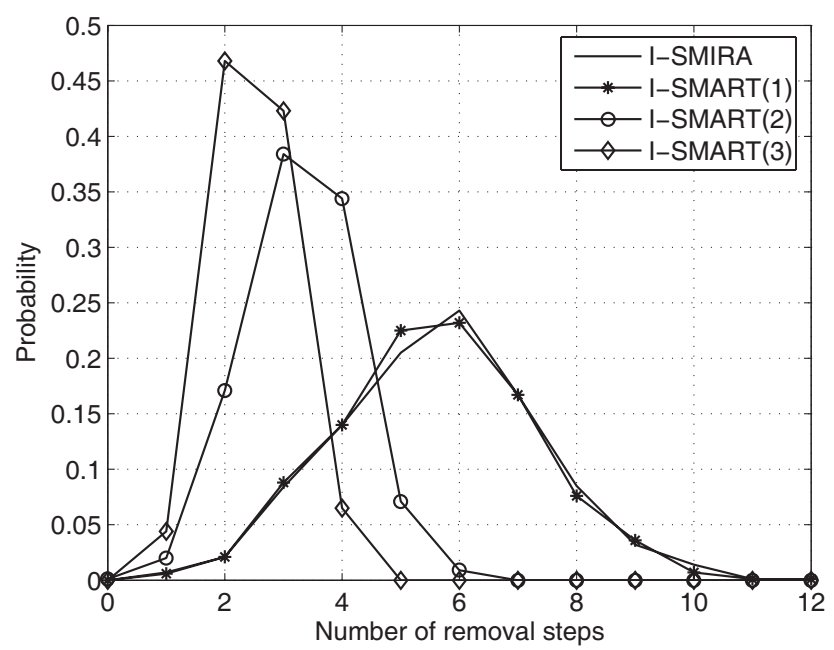

Fig. 6. Probability distribution for the number of removal steps under different removal algorithms (for $N=15, I=5 N_{0}$, SINR $=15 \mathrm{~dB}$ ).

between outage performance and the speed of removal, the probability distribution of the number of removal steps is shown in Fig. 6 for different removal algorithms. It is evident that the number of removal steps tends to decrease rapidly with $R$ (i.e., the number of removed links in each removal step) for the I-SMART(R) algorithm. Therefore, while it may be desirable to adopt single removal algorithms in slow fading environments to achieve good outage performance, multiple removal algorithms may be employed in scenarios where the channel experiences fast fading.

When the network load is low and all requesting links can be admitted while satisfying both QoS and interference constraints, we perform the joint rate and power allocation as presented in Section IV. In Fig. 7, we show the total throughput versus the minimum processing gain under different sets of constraints for both max-min and proportional fairness criteria. As expected, the more stringent the QoS and the interference constraints are, the lower is the throughput that can be achieved. This figure also shows that joint rate and power allocation with PF criterion achieves significantly

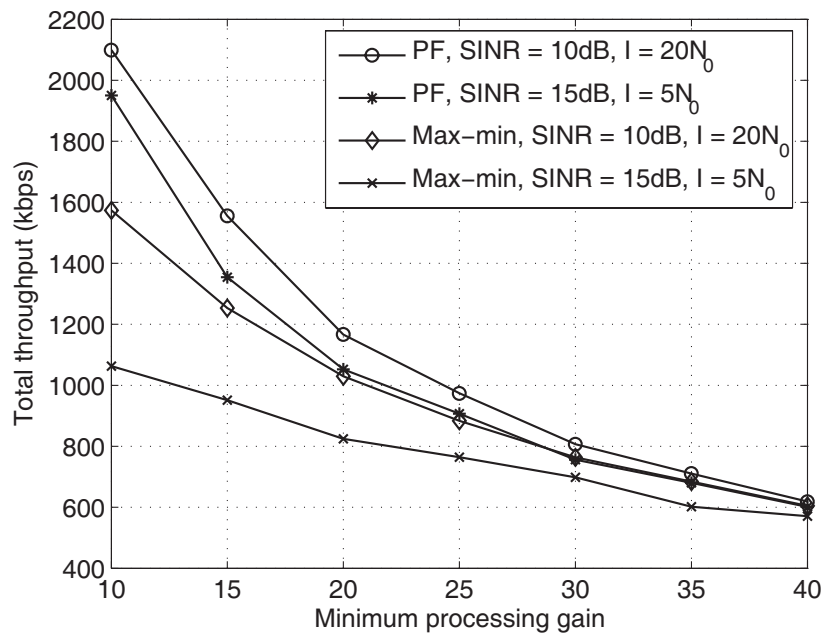

Fig. 7. Total throughput under different fairness criteria (for $N=5$ ).

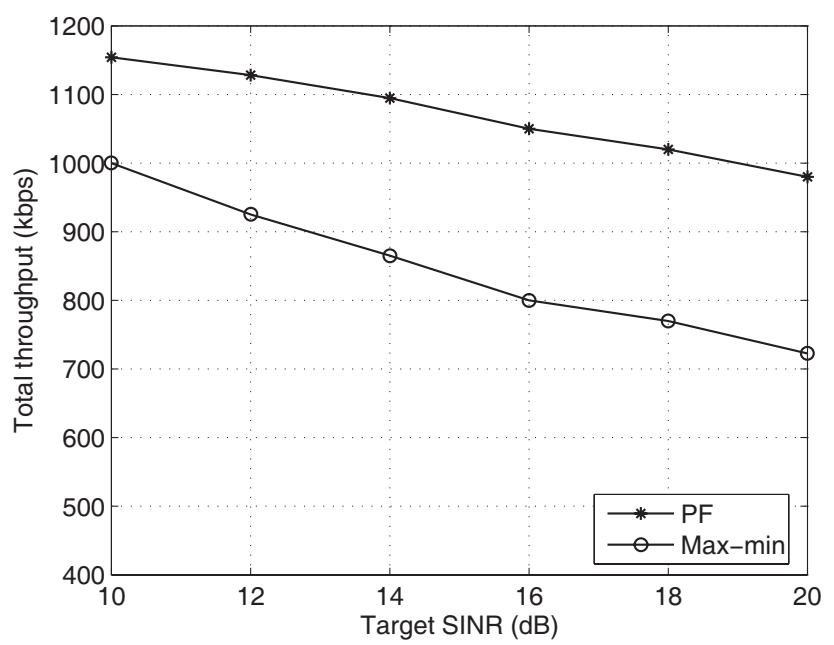

Fig. 8. Total throughput versus target SINR under different fairness criteria (for $R_{i}^{\min }=256 \mathrm{Kbps}, N=5, I=5 N_{0}$ ).

higher throughput than that with max-min fairness criterion. In addition, when the minimum processing gain increases, the differences among total throughput under different fairness criteria and different sets of QoS and interference constraints become smaller. This is because, when the minimum processing gain increases, the maximum rate decreases which essentially reduces the feasible region. Therefore, the gaps among different throughput curves become smaller.

Throughput performance under max-min and PF criteria versus the target SINR values is illustrated in Fig. 8. Again, it is observed that rate and power allocation under PF criterion consistently achieves higher throughput than that under max-min fairness criterion. Recall that rate/power allocation under max-min fairness achieves perfectly fair rates for all links. To show the fairness behavior for the PF case, we plot its fairness index versus minimum processing gain in Fig. 9. Here, the fairness index is calculated as $F_{I}=\left(\sum_{i=1}^{N} R_{i}\right)^{2} /\left(N \sum_{i=1}^{N} R_{i}^{2}\right)$ [24] which becomes closer to one as the rate allocation becomes fairer. Fig. 9 shows that rate/power allocation under PF criterion is not very bad in maintaining fairness for different links. Also, fairness 


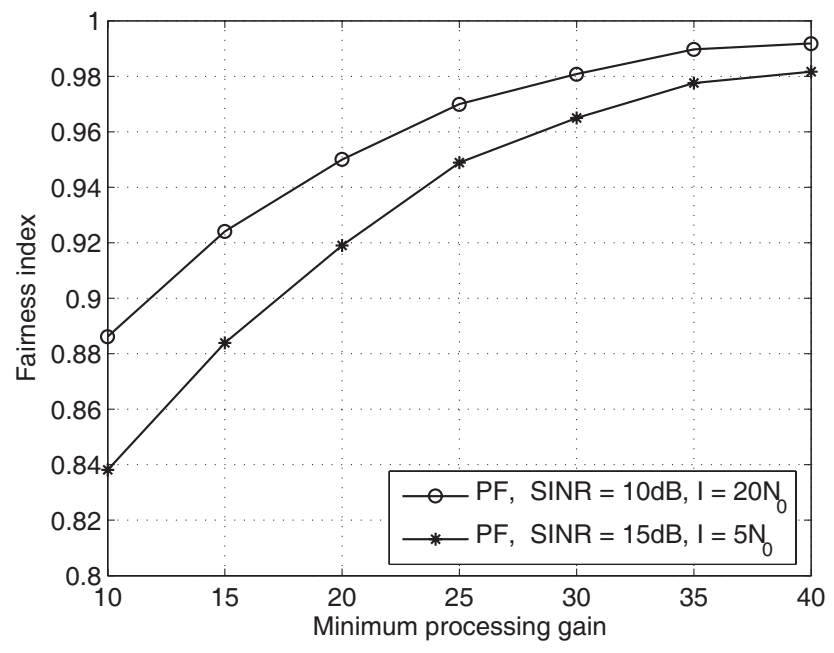

Fig. 9. Fairness index under proportional fairness (for $N=5$ ).

improves when the minimum processing gain increases (i.e., smaller feasible rate region) and/or QoS and interference constraints are less stringent. Results from Fig. 7-9, therefore, suggest that the PF criterion may be chosen for rate/power allocation to achieve better throughput if some degree of unfairness can be tolerated by different secondary links.

\section{Conclusions}

We have presented a framework to solve the spectrum sharing problem in cognitive wireless networks with spectrum underlay. In particular, admission control algorithms for spectrum underlay in CDMA networks have been proposed which aim to remove the least number of secondary links such that both the QoS constraints in terms of target SINR for accepted links and the interference constraints for primary links are satisfied. Then, we have formulated the joint rate and power allocation problems for the secondary links as optimization problems with both QoS and interference constraints under low network load conditions. We have shown how to transform these optimization problems into geometric programs in the convex form so that the globally optimal solutions can be obtained. Numerical results have revealed the impacts of different system, QoS and interference constraint parameters on the network performance.

\section{APPENDiX A}

\section{PROOF OF PROPOSITION 1}

It was shown in [17] that the admission control with only QoS constraint as presented in Section III.A is NPhard. The admission control with both QoS and interference constraints includes that with only QoS constraint as a special case. Specifically, the admission control with both QoS and interference constraints degenerates into that with only QoS constraint in [17] if the interference limits in (3) becomes infinity (i.e., $I_{j} \rightarrow \infty$ for $j=1,2, \cdots, M$ ). Therefore, it is also NP-hard.

\section{APPENDIX B}

\section{PROOF OF PROPOSITION 2}

The removal metric of the SMART(R) algorithm can be written as

$$
\begin{aligned}
D^{\Omega}\left(P^{\Omega}\right)-D^{\Omega_{0}}\left(P^{\Omega / \Omega_{0}}\right) \\
=\sum_{i \in \Omega} \beta_{i}\left(P^{\Omega}\right)-\sum_{i \in \Omega_{0}} \beta_{i}\left(P^{\Omega / \Omega_{0}}\right) \\
=\sum_{i \notin \Omega_{0}} \beta_{i}\left(P^{\Omega}\right)+\left\{\sum_{i \in \Omega_{0}} \beta_{i}\left(P^{\Omega}\right)-\sum_{i \in \Omega_{0}} \beta_{i}\left(P^{\Omega / \Omega_{0}}\right)\right\} \\
=\sum_{i \notin \Omega_{0}} \beta_{i}\left(P^{\Omega}\right)+\left\{\sum_{i \in \Omega_{0}}\left[\sum_{j \in \Omega, j \neq i} g_{i, j}^{(s)} P_{j}^{\Omega}+N_{i}-\frac{g_{i, i}^{(s)}}{\gamma_{i}} \frac{B}{R_{i}} P_{i}^{\Omega}\right]\right. \\
\left.-\sum_{i \in \Omega_{0}}\left[\sum_{j \in \Omega_{0}, j \neq i} g_{i, j}^{(s)} P_{j}^{\Omega}+N_{i}-\frac{g_{i, i}^{(s)}}{\gamma_{i}} \frac{B}{R_{i}} P_{i}^{\Omega}\right]\right\} \\
=\sum_{i \notin \Omega_{0}} \beta_{i}\left(P^{\Omega}\right)+\sum_{i \in \Omega_{0}}\left[\sum_{j \in \Omega, j \neq i} g_{i, j}^{(s)} P_{j}^{\Omega}-\sum_{j \in \Omega_{0}, j \neq i} g_{i, j}^{(s)} P_{j}^{\Omega}\right] \\
=\sum_{i \notin \Omega_{0}} \beta_{i}\left(P^{\Omega}\right)+\sum_{i \in \Omega_{0}} \sum_{j \notin \Omega_{0}, j \neq i} g_{i, j}^{(s)} P_{j}^{\Omega} \\
=\sum_{i \notin \Omega_{0}} \beta_{i}\left(P^{\Omega}\right)+\left[\sum_{i \in \Omega} \sum_{j \notin \Omega_{0}, j \neq i} g_{i, j}^{(s)} P_{j}^{\Omega}\right. \\
\left.-\sum_{i \notin \Omega_{0}} \sum_{j \notin \Omega_{0}, j \neq i} g_{i, j}^{(s)} P_{j}^{\Omega}\right] \\
=\sum_{i \notin \Omega_{0}}\left[\sum_{i \notin \Omega_{0}} \sum_{j \notin \Omega_{0}, j \neq i} g_{i, j}^{(s)} P_{j}^{\Omega}\right]
\end{aligned}
$$

Here, equation (24) results immediately from the definition in (12). Equation (25) is obtained from equation (24) simply by splitting the first term in (24) into the first two terms in (25). Equation (26) is again due to the definition of $\beta_{i}\left(P^{\Omega}\right)$ in (11). Finally, equation (30) is obtained by applying the definition of $\alpha_{i}\left(P^{\Omega}\right)$ to the second term in equation (29). Thus, the proposition is proved.

\section{APPENDIX C \\ PROOF OF PROPOSITION 3}

The proof can be done using the procedure similar to that for proposition 3 in [19] although the objective function and the set of constraints of the optimization problem here is different from that in [19]. Specifically, we will prove the proposition by contradiction using the objective function $\min \left\{\max _{i} 1 / R_{i}\right\}$. Now, let $P^{*}$ and $R^{*}$ be the optimal solutions of the optimization problem. Then, we should have $\max _{i} 1 / R_{i}^{*} \leq \max _{i} 1 / R_{i}$ for any feasible $R$. Suppose that $\max _{i} 1 / R_{i}^{*}>\min _{i} 1 / R_{i}^{*}$ (i.e., optimal rates are not equal). Assume that link $m$ achieves the minimum, i.e., $m=\operatorname{argmin}_{i} 1 / R_{i}^{*}$. We will rewrite the SINR constraints in (21) as follows:

$$
\frac{g_{i, i}^{(s)} P_{i}}{\sum_{j=1, j \neq i}^{N} g_{i, j}^{(s)} P_{j}+N_{i}} \geq \frac{R_{i} \gamma_{i}}{B}, \quad i=1,2, \cdots, N .
$$

We observe that in the left hand side of (31) the numerator is a strictly increasing function of $P_{i}$ and the denominator is a 
strictly decreasing function of $P_{j}, j \neq i$. Therefore, there must be some power decrement $\Delta P$ and rate decrement $\Delta R$ for link $m$ and a small rate increment $\Delta R_{1}$ for all other links such that the constraints in (31) still hold. Also, with this small power decrement for link $m$, the interference constraints in (21) continue to hold. Specifically, the following constraints hold

$$
\begin{gathered}
\frac{g_{m, m}^{(s)}\left(P_{m}^{*}-\Delta P\right)}{\sum_{j=1, j \neq m}^{N} g_{m, j}^{(s)} P_{j}^{*}+N_{m}} \geq \frac{\left(R_{m}^{*}-\Delta R\right) \gamma_{m}}{B} \\
\frac{g_{i, i}^{(s)} P_{i}^{*}}{\sum_{j=1, j \neq i, j \neq m}^{N} g_{i, j}^{(s)} P_{j}^{*}+g_{i, m}^{(s)}\left(P_{m}^{*}-\Delta P\right)+N_{i}} \\
\geq \frac{\left(R_{i}^{*}+\Delta R_{1}\right) \gamma_{i}}{B}, \quad i \neq m \\
\sum_{i=1, i \neq m}^{N} g_{j, i}^{(p)} P_{i}^{*}+g_{j, m}^{(p)}\left(P_{m}^{*}-\Delta P\right) \leq I_{j}, \quad j=1,2, \cdots, M .
\end{gathered}
$$

Suppose we choose the rate and power variation small enough such that

$$
1 /\left(R_{m}^{*}-\Delta R\right) \leq \max _{i \neq m} 1 /\left(R_{i}^{*}+\Delta R_{1}\right) .
$$

Under such small rate and power variations, we have

$$
\begin{array}{r}
\max _{i}\left\{\frac{1}{R_{i}^{*}}\right\}=\max _{i \neq m}\left\{\frac{1}{R_{i}^{*}}\right\}>\max _{i \neq m}\left\{\frac{1}{R_{i}^{*}+\Delta R}\right\} \\
=\max \left\{\max _{i \neq m}\left\{\frac{1}{R_{i}^{*}+\Delta R}\right\}, \frac{1}{R_{m}^{*}-\Delta R}\right\} .
\end{array}
$$

This contradicts with the condition $\max _{i} 1 / R_{i}^{*} \leq$ $\max _{i} 1 / R_{i}$ for any feasible $R$. Therefore, we must have $\max _{i} 1 / R_{i}^{*}=\min _{i} 1 / R_{i}^{*}$.

\section{REFERENCES}

[1] FCC, Spectrum policy task force report, FCC 02-155, Nov. 2002.

[2] FCC, Facilitating opportunities for flexible, efficient, and reliable spectrum use employing cognitive radio technologies, notice of proposed rule making and order, FCC 03-322, Dec. 2003.

[3] Q. Zhao and B. M. Sadler, "A survey of dynamic spectrum access: Signal processing, networking, and regulatory policy," IEEE Signal Processing Mag., pp. 79-89, May 2007.

[4] L. B. Le, E. Hossain, and A. S. Alfa, "Delay statistics and throughput performance for multi-rate wireless networks under multiuser diversity," IEEE Trans. Wireless Commun., vol. 5, no. 11, pp. 3234-3243, Nov. 2006.

[5] L. Le and E. Hossain, "Multihop cellular networks: Potential gains, research challenges, and a resource allocation framework," IEEE Commun. Mag., vol. 45, no. 9, pp. 66-73, Sept. 2007.

[6] L. Le and E. Hossain, "Cross-layer optimization frameworks for multihop wireless networks using cooperative diversity," IEEE Trans. Wireless Commun., vol. 7, no. 7, pp. 2592-2602, July 2008.

[7] M. Oner and F. Jondral, "On the extraction of the channel allocation information in spectrum pooling systems," IEEE J. Select. Areas Commun., vol. 25, no. 3, pp. 558-565, Apr. 2007.

[8] T. Banerjee, C. Ghosh, and D. P. Agrawal, "Wireless sensor based dynamic channel selection in cellular communication by cognitive radio approach," in Proc. 1st International Conference on Cognitive Radio Oriented Wireless Networks and Communications, June 2006.

[9] C. T. Chou, S. Shankar. N, H. Kim, and K. G. Shin, "What and how much to gain by spectrum agile?" IEEE J. Select. Areas Commun., vol. 25, no. 3, pp. 576-588, Apr. 2007.

[10] A. R. Fattahi, F. Fu, M. v. d. Schaar, and F. Paganini, "Mechanismbased resource allocation for multimedia transmission over spectrum agile wireless networks," IEEE J. Select. Areas Commun., vol. 25, no. 3, pp. 601-612, Apr. 2007.
[11] H. Zheng and C. Peng, "Collaborative and fairness in opportunistic spectrum access," in Proc. IEEE ICC'05.

[12] N. Nie and C. Comaniciu, "Adaptive channel allocation spectrum etiquette for cognitive radio networks," in Proc. IEEE DySPAN'05.

[13] A. T. Hoang and Y. -C. Liang, "A two-phase channel and power allocation scheme for cognitive radio networks," in Proc. IEEE PIMRC'06.

[14] Y. Xing, C. N. Mathur, M. A. Haleem, R. Chandramouli, and K. P. Subbalakshmi, "Dynamic spectrum access with QoS and interference temperature constraints," IEEE Trans. Mobile Comp., vol. 6, no. 4, pp. 423-433, Apr. 2007

[15] F. P. Kelly, A. Maulloo, and D. Tan, "Rate control for communication networks: Shadowing prices, proportional fairness, and stability," J. Oper. Res. Soc. , vol. 49, no. 3, pp. 237-252, Mar. 1998.

[16] J. Mo and J. Walrand, "Fair end-to-end window-based congestion control," IEEE/ACM Trans. Networking, vol. 8, no. 5, pp. 556-567, Oct. 2000.

[17] M. Andersin, Z. Rosberg, and J. Zander, "Gradual removals in cellular PCS with constrained power control and noise," ACM/Baltzer Wireless Networks J., vol. 2, no. 1, pp. 27-43, 1996.

[18] S. A. Grandhi and J. Zander, "Constrained power control," Wireless Personal Commun., vol. 1, no. 4, 1995.

[19] A. Muqattash, M. Krunz, and T. Shu, "Performance enhancement of adaptive orthogonal modulation in wireless CDMA systems, " IEEE $J$. Select. Areas Commun., vol. 24, no. 3, pp. 565-578, Mar. 2006.

[20] D. I. Kim, E. Hossain, and V. K. Bhargava, "Downlink joint rate and power allocation in cellular multirate WCDMA systems," IEEE Trans. Wireless Commun., vol. 2, no. 1, pp. 69-80, Jan. 2003.

[21] S. A. Jafar and A. Goldsmith, "Adaptive multirate CDMA for uplink throughput maximization," IEEE Trans. Wireless Commun., vol. 2, no. 2, pp. 218-228, Mar. 2003.

[22] A. J. Weiss and B. Friedlander, "Channel estimation for DS-CDMA downlink with aperiodic spreading codes," IEEE Trans. Commun., vol. 47, no. 10, pp. 1561-1569, Oct. 1999.

[23] G. Chen, X. -H. Yu, and J. Wang, "Adaptive channel estimation and dedicated pilot power adjustment based on the fading-rate measurement for a pilot-aided CDMA system," IEEE J. Select. Areas Commun., vol. 19, no. 1, Jan. 2001.

[24] R. Jain, A. Durresi, and G. Babic, "Throughput fairness index: An explanation," ATM Forum Document Number: ATM Forum/990045, Feb. 1999.

[25] S. Boyd and L. Vandenberge, Convex Optimization. Cambridge University Press, 2004.

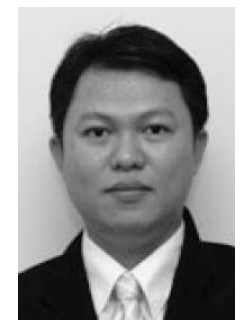

Long Bao Le (S'04-M'07) received the B.Eng. degree with highest distinction from Ho Chi Minh City University of Technology, Vietnam, in 1999, the M.Eng. degree from Asian Institute of Technology (AIT), Thailand, in 2002 and the Ph.D. degree from University of Manitoba, Canada, in 2007. He is currently a Postdoctoral Research Associate at Massachusetts Institute of Technology, USA. His current research interests include cognitive radio, link and transport layer protocol issues, cooperative diversity and relay networks, stochastic control and cross-layer design for communication networks.

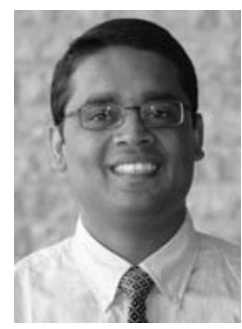

Ekram Hossain (S'98-M'01-SM'06) is currently an Associate Professor in the Department of Electrical and Computer Engineering at University of Manitoba, Winnipeg, Canada. Dr. Hossain's current research interests include design, analysis, and optimization of wireless communication networks and cognitive radio systems. He is a co-author/editor/coeditor for the books Dynamic Spectrum Access and Management in Cognitive Radio Networks (Cambridge University Press, 2009), Heterogeneous Wireless Access Networks (Springer, 2008), Introduction to Network Simulator NS2 (Springer, 2008), Cognitive Wireless Communication Networks (Springer, 2007), and Wireless Mesh Networks: Architectures and Protocols (Springer, 2007). Dr. Hossain serves as an Editor for the IEEE Transactions on Mobile Computing, the IEEE Transactions on WirELESS COMMUNICATIONS, the IEEE TRANSACTIONS ON VEHICULAR TECHNOLOGY, IEEE WiRELESS COMMUNICATIONS, and several other international journals. He is a registered Professional Engineer in the Province of Manitoba, Canada. 